\title{
Et si les cas de plagiat et de tricherie étaient liés à la nature des activités d'évaluation?
}

\section{What if cases of plagiarism and cheating related to the nature of assessment tasks?}

\section{¿Y si los casos de plagio y fraude estuvieran relacionados con la naturaleza de las actividades de evaluación?}

https://doi.org/10.52358/mm.vi9.265

Marie-Hélène Hébert, professeure

Université TÉLUQ, Canada

marie-helene.hebert@teluq.ca

Sylvie Fontaine, professeure retraitée

Université du Québec en Outaouais, Canada

sylvie.fontaine@uqo.ca

RÉSUMÉ

La pandémie de COVID-19 et la fermeture des établissements d'enseignement qu'elle a entrainée ont conduit le personnel enseignant à s'interroger sur la manière d'apprécier les apprentissages des apprenants à distance. Dans cet article, nous examinons la voie de l'évaluation authentique pour proposer aux apprenants des activités d'évaluation qui les amènent à faire usage de créativité et, ce faisant, à diminuer les cas de plagiat et de tricherie.

Mots-clés : évaluation authentique, plagiat, tricherie 
The COVID-19 pandemic and the resulting closure of educational institutions have led teachers to question how to assess students' performance in distance learning. In this article, we examine the authentic assessment pathway $t$ to propose assessment tasks to students that lead them to use creativity and decrease instances of plagiarism and cheating.

Keywords: authentic assessment, plagiarism, cheating

RESUMEN

La pandemia de COVID-19 y el consiguiente cierre de centros educativos ha llevado a los profesores a cuestionarse cómo evaluar a distancia el aprendizaje de los estudiantes. En este artículo, examinamos la vía de la evaluación auténtica para proponer a los estudiantes actividades de evaluación que los animen a utilizar la creatividad y, de este modo, reducir la incidencia del plagio y el fraude.

Palabras clave: evaluación auténtica, plagio, fraude

La pandémie de COVID-19 depuis mars 2020 a apporté son lot de transformations à l'école, et ce, de l'éducation préscolaire à l'enseignement universitaire. Au plus fort de la pandémie, avec les établissements d'enseignement fermés, le personnel enseignant a dû user d'astuces pour faire l'école à distance, intégrant des séances d'enseignement synchrones et asynchrones, avec tout ce que cela implique en termes de planification pédagogique, de production de matériels, de soutien à l'apprentissage et autres. Si chacun des gestes professionnels du personnel enseignant a dû être pensé, analysé et exécuté à la hâte dans l'urgence du moment, une question a semblé en prendre plus d'un au dépourvu : comment, au juste, apprécier les apprentissages des apprenants à distance au moment où l'épreuve papier-crayon, qui avait jusque-là dominé dans les salles de classe, ne devenait plus possible?

Pour aider le personnel enseignant à trancher, l'un des enjeux qui se posent est celui lié à la possibilité pour les apprenants de plagier ou de tricher. Soulignons que les cas de fraude (tricherie et plagiat) remettent en question la crédibilité des notes et des diplômes décernés. Or, pendant la pandémie de COVID-19, les médias ont révélé des statistiques alarmantes laissant entrevoir un accroissement des cas de fraude avec des titres comme " Hausse préoccupante du plagiat » (Poiré, 2020) ou " La tricherie, un effet secondaire de la pandémie pour les universités » (Radio-Canada, 2020).

Pourtant, en 2017, Tolman rapportait des taux de fraude tout au plus similaires entre les cours offerts à distance et ceux offerts en présence. On peut donc se poser la question suivante : que s'est-il passé entre les taux rapportés par Tolman en 2017 et les statistiques alarmantes d'aujourd'hui? À la façon de Papi, Hébert et Gérin-Lajoie (sous presse), nous posons l'hypothèse que, dans plusieurs établissements d'enseignement, "l'augmentation des cas de plagiat ou tricherie pendant la pandémie est liée à la transposition de types d'évaluation conçus pour un mode présentiel, mais réalisés à distance en raison de la situation $"$. 
En mode présentiel, il est courant d'administrer aux apprenants des tâches à choix multiple ou à réponses courtes qui font appel aux connaissances emmagasinées par ces derniers. Ces tâches sont habituellement administrées en salle de classe, sous surveillance et sans possibilité de recours aux manuels scolaires ou autres outils. Lorsqu'une pandémie oblige la fermeture des établissements d'enseignement, il devient risqué d'offrir ce même type d'activité d'évaluation à réaliser à la maison où tout devient à la portée des apprenants : le manuel obligatoire, les notes de cours, Internet, l'aide d'un pair ou autres.

Si la littérature scientifique et l'expertise pratique foisonnent quant aux manières d'améliorer l'efficacité des épreuves papier-crayon administrées sous surveillance en salle de classe, à quoi peut ressembler une activité d'évaluation conçue pour être administrée à distance? En tant que professeures en mesure et évaluation des apprentissages qui avons enseigné pendant plusieurs années les fondements de la discipline aux futurs enseignants, nous proposons que l'une des voies à suivre pour diminuer les cas de plagiat et de tricherie est celle d'administrer aux apprenants des activités d'évaluation authentiques (Wiggins, 1993) qui les amènent à faire preuve de créativité dans leurs réponses.

Des premiers travaux de Grant Wiggins à aujourd'hui, plusieurs auteurs (p. ex. : Duval et Pagé, 2013; Leroux, Hébert et Paquin, 2015; Louis, 2004) ont mis en relief différentes caractéristiques de ces activités d'évaluation authentiques. Ainsi, ces activités s'inspirent du quotidien des apprenants ou du milieu de travail futur dans lequel ils évolueront, elles dépassent la simple redite de connaissances en exigeant la combinaison originale de plusieurs savoirs, savoir-faire et savoir-être, elles conduisent chacun des apprenants à produire une réponse qui lui est propre, voire unique, et leur offrent un certain contrôle sur la manière d'y répondre. Les activités d'évaluation authentiques sont par ailleurs réputées pour motiver davantage les apprenants que les tâches dites scolaires comme les questions à choix multiple et à réponses courtes. Elles s'apprécient par le jugement du personnel enseignant, jugement qui s'appuie sur des critères d'évaluation préalablement définis.

À titre d'exemples, nous partageons ci-dessous quelques activités d'évaluation authentiques qui pourraient être administrées aux étudiants des cours d'évaluation des apprentissages que nous offrons à l'université. Ces activités d'évaluation, qui pourraient remplacer le traditionnel examen écrit à correction objective, sont en lien avec l'objectif même de ces cours qui visent à développer chez les étudiants la compétence à évaluer les apprentissages des élèves afin de vérifier la progression et l'acquisition des compétences, de communiquer clairement les résultats de l'évaluation et d'adapter l'enseignement en conséquence.

\section{Exemples d'activités d'évaluation authentiques}

- Inviter les apprenants à contacter un enseignant du primaire ou du secondaire dans le but de concevoir un examen sur une partie de matière donnée qui sera par la suite réalisé dans la classe de l'enseignant;

- Faire écrire aux apprenants une lettre adressée au ministre de l'Éducation afin de lui proposer des modifications pour mieux intégrer l'évaluation dans les classes du primaire ou du secondaire en contexte de pandémie;

- Inviter les apprenants à concevoir un aide-mémoire visuel (affiche) sur les diverses manières de faire l'évaluation dans les classes du primaire ou du secondaire;

- Faire réaliser par les apprenants une capsule d'information à l'intention des parents des élèves du primaire ou du secondaire pour leur expliquer la manière dont l'évaluation se fera dans la classe de leur enfant; 
- Amener les apprenants à écrire le scénario d'une rencontre avec un parent dans le cadre de la remise des bulletins au primaire ou au secondaire;

- Amener les apprenants à enregistrer un balado pour faire connaitre à des enseignants des manières innovantes de pratiquer l'évaluation en soutien à l'apprentissage auprès des élèves du primaire ou du secondaire;

- Faire réaliser par les apprenants un tutoriel vidéo pour soutenir l'utilisation d'une plateforme qui permet la conception de quiz interactifs pour apprécier les apprentissages des élèves du primaire ou du secondaire;

- Faire concevoir par les apprenants une activité d'évaluation authentique qui pourrait servir à apprécier les apprentissages des étudiants qui suivront le cours l'an prochain.

En somme, nous souhaitions rappeler par ce court article que pour diminuer le plagiat et la tricherie, il faut voir plus loin que simplement transposer les évaluations conçues pour un mode présentiel en format numérique. Pour y parvenir, l'une des voies à emprunter réside du côté des activités d'évaluation authentiques qui offrent aux apprenants de faire la démonstration de leurs apprentissages dans des tâches réalistes et qui ont du sens pour eux, tout en leur permettant d'avoir accès à tout leur matériel, à Internet, à leurs pairs, etc. En cela, les activités d'évaluation authentiques sont le reflet de ce que sera leur réalité dans leur vie professionnelle future. Évidemment, il n'y a pas de garantie que les productions des apprenants seront exemptes de plagiat ou de tricherie, mais accorder de la valeur à leur créativité, leur donner un certain contrôle sur l'activité d'évaluation et la rendre pertinente pour leur futur contexte professionnel pourra possiblement susciter davantage leur engagement réel et ainsi diminuer la fraude scolaire.

\section{Liste de références}

Duval, A.-M. et Pagé, M. (2013). La situation authentique : de la conception à l'évaluation. AQPC.

Leroux, J. L., Hébert, A. et Paquin, J. (2015). Concevoir des tâches d'évaluation en situation authentique. Dans J. L. Leroux (dir.), Évaluer les compétences au collégial et à l'université : un guide pratique. AQPC.

Louis, R. (2004). L'évaluation des apprentissages en classe : théorie et pratique. Beauchemin.

Papi, C., Hébert, M.-H. et Gérin-Lajoie, S. (sous presse). La formation à distance : une source d'inspiration pour repenser l'évaluation? Actes du colloque ADMÉÉ-Europe 2022.

Poiré, A.-S. (2020, 15 novembre). Hausse préoccupante du plagiat. Le Journal de Montréal. https://www.journaldemontreal.com/2020/11/15/hausse-preoccupante-du-plagiat

Radio-Canada (2020, 21 juin). La tricherie, un effet secondaire de la pandémie pour les universités. Radio-Canada. https://ici.radio-canada.ca/nouvelle/1714027/plagiat-cours-en-ligne-surveillance

Tolman, S. (2017). Academic dishonesty in online courses: considerations for graduate preparatory programs in higher education. College Student Journal, 51(4), 579-584. https://tinyurl.com/mvuuum7f

Wiggins, G. P. (1993). Assessing student performance: exploring the purpose and limits of testing. Jossey-Bass. 